\title{
Fetal Testosterone Influences Sexually Dimorphic Gray Matter in the Human Brain
}

\author{
Michael V. Lombardo, ${ }^{1}$ Emma Ashwin, ${ }^{1,2}$ Bonnie Auyeung, ${ }^{1}$ Bhismadev Chakrabarti, ${ }^{1,3}$ Kevin Taylor, ${ }^{4}$ Gerald Hackett, ${ }^{5}$ \\ Edward T. Bullmore, ${ }^{6}$ and Simon Baron-Cohen ${ }^{1}$ \\ ${ }^{1}$ Autism Research Centre, Department of Psychiatry, University of Cambridge, Cambridge CB2 8AH, United Kingdom, ${ }^{2}$ Department of Psychology, \\ University of Bath, Bath BA2 7AY, United Kingdom, ${ }^{3}$ Centre for Integrative Neuroscience and Neurodynamics, School of Psychology and Clinical Language \\ Sciences, University of Reading, Reading RG6 6AH, United Kingdom, ${ }^{4}$ Department of Clinical Biochemistry, Addenbrooke's Hospital, Cambridge CB2 0QQ, \\ United Kingdom, ${ }^{5}$ Department of Fetal Medicine, Rosie Maternity Hospital, Cambridge CB2 0SW, United Kingdom, and ${ }^{6}$ Brain Mapping Unit, Department \\ of Psychiatry, University of Cambridge, Cambridge CB2 0SZ, United Kingdom
}

In nonhuman species, testosterone is known to have permanent organizing effects early in life that predict later expression of sex differences in brain and behavior. However, in humans, it is still unknown whether such mechanisms have organizing effects on neural sexual dimorphism. In human males, we show that variation in fetal testosterone (FT) predicts later local gray matter volume of specific brain regions in a direction that is congruent with sexual dimorphism observed in a large independent sample of age-matched males and females from the NIH Pediatric MRI Data Repository. Right temporoparietal junction/posterior superior temporal sulcus (RTPJ/pSTS), planum temporale/parietal operculum (PT/PO), and posterior lateral orbitofrontal cortex (plOFC) had local gray matter volume that was both sexually dimorphic and predicted in a congruent direction by FT. That is, gray matter volume in RTPJ/pSTS was greater for males compared to females and was positively predicted by FT. Conversely, gray matter volume in PT/PO and plOFC was greater in females compared to males and was negatively predicted by FT. Subregions of both amygdala and hypothalamus were also sexually dimorphic in the direction of Male $>$ Female, but were not predicted by FT. However, FT positively predicted gray matter volume of a non-sexually dimorphic subregion of the amygdala. These results bridge a long-standing gap between human and nonhuman species by showing that FT acts as an organizing mechanism for the development of regional sexual dimorphism in the human brain.

\section{Introduction}

Males and females differ in subtle ways and understanding the mechanisms behind such differences has been a major focus of both scientific inquiry and controversy throughout the decades. Two biological factors are important for understanding sex dif-

Received Aug. 26, 2011; revised 0ct. 24, 2011; accepted Nov. 1, 2011.

Author contributions: E.A., G.H., and S.B.-C. designed research; E.A. and B.A. performed research; M.V.L. and K.T. contributed unpublished reagents/analytic tools; M.V.L. analyzed data; M.V.L., B.A., B.C., E.T.B., and S.B.-C. wrote the paper.

This work was supported by grants from the Wellcome Trust and the MRC (to S.B.-C. and E.T.B.) and was conducted in association with the NIHRCLAHRC for Cambridgeshire and Peterborough Foundation National Health Services Trust. M.V.L., B.C., and B.A. were supported by Research Fellowships from Jesus, Darwin, and Wolfson Colleges, respectively. FT assays were made possible by a grant to S.B.-C. from the Nancy Lurie Marks Family Foundation. We thank John Suckling, MengChuan Lai, Amber Ruigrok, and Rebecca Knickmeyer forvaluable comments and discussions. Wealso thank the families who have taken part in this longitudinal study. Finally, we thank the NIH Pediatric MRI Data Repository created by the NIH MRI Study of Normal Brain Development. This is a multisite, longitudinal study of typically developing children, from newborns to young adults, conducted by the Brain Development Cooperative Group and supported by the National Institute of Child Health and Human Development, the National Institute on Drug Abuse, the National Institute of Mental Health, and the National Institute of Neurological Disorders and Stroke (Contract numbers N01-HD02-3343, N01-MH9-0002, and N01-NS$9-2314,-2315,-2316,-2317,-2319$, and -2320$)$. A listing of the participating sites and a complete listing of the study investigators can be found at https://nihpd.crbs.ucsd.edu/nihpd/info/participating_centers.html.

This manuscript reflects the views of the authors and may not reflect the opinions or views of the Brain Development Cooperative Group Investigators or the NIH.

E.T.B. is half-time used by the University of Cambridge and half-time by GlaxoSmithKline. The other authors declare no competing financial interests.

This article is freely available online through the J Neurosci Open Choice option.

Correspondence should be addressed to either Michael V. Lombardo or Simon Baron-Cohen, Autism Research Centre, Douglas House, 18B Trumpington Road, Cambridge CB2 8AH, UK. E-mail: ml437@cam.ac.uk or sb205@cam.ac.uk.

DOl:10.1523/JNEUROSCI.4389-11.2012

Copyright $\odot 2012$ the authors $\quad 0270-6474 / 12 / 320674-07 \$ 15.00 / 0$ ferences: sex hormones and sex chromosome effects (McCarthy and Arnold, 2011). Early exposure to sex steroids plays a major organizing influence on the expression of later sexual dimorphism (Phoenix et al., 1959; Arnold and Breedlove, 1985; Arnold, 2009). Sex steroids influence a variety of cellular processes, including gene expression, and are ideal candidates for exerting epigenetic effects on the sexually dimorphic developing brain (McCarthy et al., 2009). Sex chromosomes also exert an important additional influence on sexual differentiation. Both the $\mathrm{X}$ and $\mathrm{Y}$ chromosomes possess many genes that are expressed differentially in male and female brains (Reinius and Jazin, 2009; McCarthy and Arnold, 2011). Transgenic techniques that dissociate sex hormones from sex chromosome effects have shown independent contributions of each factor on the development sexually dimorphic neural phenotypes (De Vries et al., 2002), though the two likely interact (McCarthy et al., 2009; Paus et al., 2010; Raznahan et al., 2010; McCarthy and Arnold, 2011).

In contrast to studying sex differences in nonhuman species, many ethical, methodological, and practical challenges are raised in relation to testing such questions in humans (Baron-Cohen et al., 2004). Standard experimental manipulations such as castration, androgen blockage, and transgenic models are obviously unethical in humans. Thus, a major gap remains in understanding the mechanisms contributing to sex differences in the human brain. Bridging this gap is important not only for understanding basic questions about why males and females differ, but also for 


\begin{tabular}{|c|c|c|c|c|c|c|}
\hline Region & Hemisphere & BA & $\operatorname{MNI}(x, y, z)$ & $\begin{array}{l}t \\
\text { value }\end{array}$ & $\begin{array}{l}\text { Cluster } \\
\text { size }\end{array}$ & $p$ value \\
\hline \multicolumn{7}{|l|}{ Positive correlation } \\
\hline Postcentral gyrus & L & 3 & $-43,-29,60$ & 6.83 & 8252 & $9.99 \times 10^{-5}$ \\
\hline Postcentral gyrus & L & 3 & $-45,-25,52$ & 6.83 & & \\
\hline Precentral gyrus & L & 44 & $-39,14,40$ & 5.10 & & \\
\hline Postcentral gyrus & $\mathrm{R}$ & 3 & $45,-26,66$ & 5.68 & 7273 & $1.98 \times 10^{-4}$ \\
\hline Central sulcus & $\mathrm{R}$ & 4 & $51,-16,61$ & 5.63 & & \\
\hline Central sulcus & $\mathrm{R}$ & 6 & $46,-9,45$ & 4.08 & & \\
\hline RTPJ & $\mathrm{R}$ & 39 & $41,-64,32$ & 4.96 & 3898 & 0.045 \\
\hline RTPJ & $\mathrm{R}$ & 39 & $49,-51,29$ & 4.72 & & \\
\hline pSTS & $\mathrm{R}$ & 22 & $61,-48,23$ & 4.36 & & \\
\hline Amygdala* & L & - & $-18,-6,-27$ & 4.46 & & $0.0338^{*}$ \\
\hline \multicolumn{7}{|c|}{ Negative correlation } \\
\hline PT/PO & $\mathrm{R}$ & 48 & $49,-20,14$ & 5.71 & 18871 & $9.99 \times 10^{-5}$ \\
\hline plOFC & $\mathrm{R}$ & 48 & $26,17,-20$ & 5.69 & & \\
\hline sgACC & B & 25 & $1,20,-12$ & 5.43 & & \\
\hline Pars triangularis & R & 45 & $51,21,0$ & 3.77 & & \\
\hline Anterior insula & $\mathrm{R}$ & 47 & $32,21,-11$ & 3.72 & & \\
\hline PT/PO & L & 22 & $-66,-24,15$ & 7.90 & 14948 & $9.99 \times 10^{-5}$ \\
\hline plOFC & L & 38 & $-33,17,-20$ & 5.47 & & \\
\hline mSTS & L & 21 & $-63,-19,-8$ & 4.95 & & \\
\hline Pars triangularis & L & 47 & $-53,31,-9$ & 3.83 & & \\
\hline Anterior insula & L & 47 & $-43,23,-3$ & 3.71 & & \\
\hline
\end{tabular}

$p$ values are cluster-corrected based on extent. ${ }^{*} p<0.05$ FWE using small-volume correction. L, Left; $R$, right.

understanding possible mechanisms behind neurodevelopmental conditions with skewed sex ratios (Baron-Cohen et al., 2011).

Here we examine, for the first time, the role of fetal testosterone (FT) in organizing the human brain for expression of sexual dimorphism later in life. Using structural MRI, we scanned 28 typically developing boys (aged 8-11 years old; for participant characteristics, see Table 1) whose FT was measured from amniotic fluid samples collected during amniocentesis (13-20 weeks gestation). We used a male-only sample to eliminate potential confounds of imprinting and X-inactivation effects in females (Good et al., 2003). Using voxel-based morphometry (VBM), we examined the relationship between FT and later local gray matter (GM) volume. We then used VBM to assess sexual dimorphism in an independent age-matched sample of 101 boys and 116 girls from the NIH Pediatric MRI Data Repository (Brain Development Cooperative Group and Evans, 2006). The combination of these two datasets allowed us to localize which, if any, sexually dimorphic brain regions in a representative sample of the population are also associated with FT in a congruent direction (e.g., Male $>$ Female sexual dimorphism and FT-positive correlation; Female $>$ Male sexual dimorphism and FT-negative correlation). Furthermore, the independent NIH sample allowed us to create sexually dimorphic ROIs that we then used to estimate the magnitude for which FT predicts later local GM volume in these regions.

\section{Materials and Methods}

Participants. Participants in the FT cohort were recruited from a longitudinal study of the effects of FT on cognitive, behavioral, and brain development (Baron-Cohen et al., 2004). Initial screening consisted of reviewing medical records of patients who underwent amniocentesis in the Cambridgeshire (UK) region between 1996 and 1999. Individuals were excluded if (1) the amniocentesis revealed a chromosomal abnormality, (2) there was a twin pregnancy, (3) the pregnancy ended in termination or miscarriage, (4) relevant information was absent from the medical records, or (5) medical practitioners indicated it would be inappropriate to contact the family. Any child that presented with any devel- opmental abnormalities postnatally was excluded from testing. The final set of participants was 28 typically developing boys (mean age, 9.5 years; SD, 0.92 years; range, $8-11$ years). Twenty-two of the 28 participants $(78 \%)$ were right-handed as measured by the Edinburgh Handedness Inventory (EHI) (Oldfield, 1971). Informed consent was obtained from all the participant's legal guardians in accordance with procedures approved by the local research ethics committee.

In the analysis of sexual dimorphism, we used publicly available data from the NIH Pediatric MRI Repository (Brain Development Cooperative Group and Evans, 2006) (https://nihpd.crbs.ucsd.edu). We used participants with an MRI scan between the ages of $8-11$ years from all six centers from the Objective 1 dataset. For individuals with more than one scan across this age range, the scan chosen for inclusion in this analysis was randomly selected. Males $(n=101$; mean age, 9.51 years; SD, 1.06 years; range, $8-11$ years) and females ( $n=116$; mean age, 9.5 years; SD, 1.09 years; range, $8-11$ years $)$ did not differ in age $(t=-0.04, p=0.96)$ and did not differ in age with the FT-cohort (males: $t=-0.06, p=0.94$; females: $t=-0.03, p=0.97$ ). Handedness was assessed with eight activities modified from the EHI. For each activity, a score of 1 was used if the participant performed the action with the right hand, otherwise it was given a score of 0 . Classification of individuals as right-handed was based on a summary score across these eight items that was $\geq 7$. Eighty-six males $(85 \%)$ and 102 females (88\%) were right-handed. Sixteen males $(15 \%)$ and 14 females $(12 \%)$ were non-right handed.

Fetal testosterone collection and measurement. FT was measured from amniotic fluid samples collected between 13 and 20 weeks of gestation (mean FT, $0.79 \mathrm{nmol} / \mathrm{L} ; \mathrm{SD}, 0.34 \mathrm{nmol} / \mathrm{L}$; range, $0.25-1.70 \mathrm{nmol} / \mathrm{L}$ ). This is within the $8-24$ week period that is hypothesized to be critical for human sexual differentiation (Hines, 2004). Seven participants had missing data with regards to the sampling time of amniocentesis. However, analysis of the remaining 21 participants (mean gestational age at amniocentesis, 16.37 weeks; SD, 1.33 weeks; range, 14-19 weeks) showed no linear or quadratic relationship between gestational age at FT sampling and FT level $(r=0.12, p=0.60)$. This confirms prior work from multiple labs showing an absence of a relationship during the period typically used for amniocentesis (Grimshaw et al., 1995; Chapman et al., 2006; Sarkar et al., 2007; Auyeung et al., 2009, 2010; Bergman et al., 2010). Given the absence of a relationship between FT and gestational age at amniocentesis, we included all 28 participants in the main VBM analysis.

FT was assayed via radioimmunoassay. Amniotic fluid was extracted with diethyl ether, which was evaporated to dryness at room temperature and the extracted material redissolved in an assay buffer. Testosterone was assayed by the Count-a-Coat method (Diagnostic Product), which uses an antibody to testosterone coated onto propylene tubes and a ${ }^{125} \mathrm{I}$ labeled testosterone analog. The detection limit of the assay using the ether-extraction method is $\sim 0.05 \mathrm{nmol} / \mathrm{L}$. The coefficient of variation (CV) for between-batch imprecision is $19 \%$ at a concentration of 0.8 $\mathrm{nmol} / \mathrm{L}$ and $9.5 \%$ at a concentration of $7.3 \mathrm{nmol} / \mathrm{L}$. The CVs for withinbatch imprecision are $15 \%$ at a concentration of $0.3 \mathrm{nmol} / \mathrm{L}$ and $5.9 \%$ at a concentration of $2.5 \mathrm{nmol} / \mathrm{L}$. This method measures total extractable testosterone.

MRI acquisition and image processing. All imaging for the FT cohort took place at the Wolfson Brain Imaging Centre at Addenbrooke's Hospital (Cambridge, UK) on a Tim Trio 3 tesla magnet (Siemens Medical Solutions). High-resolution T1-weighted three-dimensional MP-RAGE structural images were acquired (slice thickness, $1 \mathrm{~mm}$; TR, $2300 \mathrm{~ms}$; TE, $2.98 \mathrm{~ms}$; field of view, $256 \times 240 \times 176 \mathrm{~mm}$; flip angle, $98^{\circ}$; voxel size, 1 $\mathrm{mm}^{3}$ isotropic). Imaging for the NIH-cohort took place across six centers in the USA. Images were obtained on $1.5 \mathrm{~T}$ systems from GE Healthcare or Siemens Medical Systems using a three-dimensional T1-weighted SPGR sequence with the following parameters: TR, 22-25 ms; TE, 10-11 ms; excitation pulse, $30^{\circ}$; refocusing pulse, $180^{\circ}$; orientation, sagittal; field of view: anterior-posterior, $256 \mathrm{~mm}$; left-right, $160-180 \mathrm{~mm}$ (whole-head coverage); voxel size, $1 \mathrm{~mm}^{3}$, where the maximum number of slices on GE scanners was 124, and hence the slice thickness was increased to $1.5 \mathrm{~mm}$. More details can be found online at the NIH Pediatric MRI Repository website (https://nihpd.crbs.ucsd.edu) (Brain Development Cooperative Group and Evans, 2006). 
T1-weighted images from both the FT and NIH cohorts were run through the exact same image-processing pipeline. This pipeline consisted of an initial segmentation in SPM8 (http://www.fil.ion.ucl.ac.uk/ $\mathrm{spm} /$ ) to get native-space gray matter (GM), white matter (WM), and cerebrospinal fluid (CSF) maps. This step used prior tissue probability maps generated from the Template-O-Matic toolbox (http://dbm.neuro. uni-jena.de/software/tom/) (Wilke et al., 2008) and set for the age range of 8-11 years. Total GM, WM, and CSF volumes were then calculated from these native-space segmentation maps for the purposes of using these total estimates as covariates in statistical analyses. Next, we used a diffeomorphic nonlinear registration tool (DARTEL) (Ashburner, 2007) to register the native-space segmented images to each other, nonlinearly warp the data to create a study-specific template, and then modulate images according to the flow fields estimated from DARTEL at a resolution of $1 \mathrm{~mm}$ isotropic voxel size. These modulated maps were then registered to MNI space and smoothed with at $4 \mathrm{~mm}$ FWHM kernel. Smoothing at this size was done to optimize the analyses to be sensitive to very small localized differences in smaller subcortical structures known to express sexual dimorphism (e.g., hypothalamus and amygdala). Furthermore, because DARTEL achieves much more accurate registration than previous warping techniques (Klein et al., 2009), registration error is reduced and requires less smoothing for correction.

Statistical analyses. Statistical analyses were performed using Tor Wager's robust regression Matlab toolbox (Wager et al., 2005). For the FT cohort, modulated GM images in MNI space were entered as the dependent variable and regressors were age, total GM volume, and FT. For the NIH cohort, the same procedure was used except center and sex were also included as regressors. For each cohort, an explicit GM mask was used. In the FT analysis, contrasts were computed for the positive and negative correlation with FT after controlling for age and total GM volume. For the sex difference analysis in the NIH cohort, contrasts were computed for Males $>$ Females and Females $>$ Males after controlling for age, total GM volume, and center. Each voxel was thresholded at $p<$ 0.025 . Correction for multiple comparisons at a corrected $p<0.05$ was achieved with cluster extent thresholding (Forman et al., 1995). To derive the minimum cluster extent threshold for ensuring a corrected $p<0.05$, we used a custom Matlab script (written by M.V.L.) that runs a Monte Carlo simulation (10,000 iterations) to estimate a null distribution of maximum cluster extent thresholds. On each iteration, we simulated smoothed whole-brain maps of values taken randomly from a Gaussian distribution, applied a $p<0.025$ voxelwise threshold, and then calculated the maximum cluster extent. From the distribution of simulated maximum cluster extents, we found the extent threshold under this distribution where $5 \%$ of cluster extents were equal to or greater than that value. This critical extent threshold (FT cohort, $k=3835$; NIH cohort, $k=3780$ ) was used as the minimum cluster extent threshold to correct for multiple comparisons at $p<0.05$. In addition to this whole-brain correction, we also used small-volume correction (SVC; $p<0.05$, FWE) for a priori anatomical masks of the amygdala and hypothalamus (Worsley et al., 1996; Friston, 1997).

To specifically assess which regions were both sexually dimorphic and associated with FT, we used a logical AND masking procedure on the whole-brain-corrected results maps to implement conjunction analyses (Nichols et al., 2005). These conjunction analyses isolate regions whose direction of the FT correlation was congruent with the direction of sexual dimorphism observed in the NIH cohort.

Finally, we estimated the magnitude of association between FT and local GM volume in particular areas noted by the conjunction analysis or within a priori regions such as amygdala and hypothalamus. ROIs for this analysis were constructed for each region based on the independent criteria of being sexually dimorphic in the NIH cohort and, where anatomical boundaries could be defined, were also located within the anatomical region defined by the Harvard-Oxford atlas in FSL (http://www.fmrib. ox.ac.uk/fsl/data/atlas-descriptions.html). Significance for all correlations were reported with one-tailed $p$ values because of the strict directionality hypotheses.

\section{Results}

\section{FT-GM volume correlation}

Increasing FT was predictive of more local GM volume (i.e., positive correlation) in right temporoparietal junction/posterior superior temporal sulcus (RTPJ/pSTS) and bilateral somatosensory, motor, and premotor cortex. Using anatomical masks of the amygdala and hypothalamus as small volumes of interest, only a ventral medial subregion in the left amygdala was positively predicted by FT (MNI: $\left.x=-18, y=-6, z=-27 ; t=4.46, p_{\mathrm{SVC}-\mathrm{FWE}}=0.0338\right)$. Using the Jülich cytoarchitectonic atlas in FSL (http://www.fmrib.ox.ac.uk/fsl/ data/atlas-descriptions.html) (Amunts et al., 2005) for labeling, this subregion was located within the superficial subdivision consisting of the anterior amygdaloid area, amygdalopyriform transition area, amygdaloid-hippocampal area, and the ventral and posterior cortical nuclei. In the other direction, increasing FT was predictive of less local GM volume (i.e., negative correlation) bilaterally along the Sylvian fissure, particularly in planum temporale/parietal operculum (PT/PO), Heschl's gyrus, posterior lateral orbitofrontal cortex (plOFC), anterior insula, pars triangularis, and left middle and superior temporal gyrus (Fig. 1A, Table 1).

\section{Sexually dimorphic GM volume}

Within the NIH cohort, Male > Female sexual dimorphism was observed in several subcortical areas such as dorsal and lateral amygdala (laterobasal, superficial, and centromedial cytoarchitectonic subdivisions), hypothalamus, putamen extending into globus pallidus, and ventral tegmental area. Within cortex, Male $>$ Female sexual dimorphism was observed in left lateral occipital cortex, bilateral superior occipital cortex, cuneus, posterior cingulate cortex/retrosplenial cortex, right inferior parietal lobule, bilateral premotor cortex, RTPJ/pSTS, bilateral posterior middle temporal gyrus, right temporal pole, left posterior middle frontal gyrus, and left anterior prefrontal cortex. In the other direction, Female $>$ Male sexual dimorphism was observed in bilateral precuneus extending into superior parietal lobule, caudal anterior and middle cingulate cortex, right $\mathrm{PT} / \mathrm{PO}$, right motor and somatosensory cortex, bilateral posterior medial and lateral orbitofrontal cortex, and anterior medial prefrontal cortex (Fig. 1B).

\section{Overlap between FT and sexual dimorphism}

Using conjunction analyses (Nichols et al., 2005), we assessed whether there was overlap between FT-correlated voxels and sexual dimorphism. Overlap between Male $>$ Female sexual dimorphism and FT-positive correlation was observed in RTPJ/pSTS and a small cluster in right dorsal premotor cortex (PMd). In the other direction, overlap between Female $>$ Male sexual dimorphism and FT-negative correlation was observed in right $\mathrm{PT} / \mathrm{PO}$ and right plOFC (Fig. 2A). With a Monte Carlo simulation (5000 iterations), we determined the probability of getting the same or greater number of overlapping voxels in randomly generated suprathreshold maps. On each iteration, we generated two smoothed whole-brain maps filled with values sampled randomly from a Gaussian distribution. These maps were thresholded at the same voxelwise and cluster extent thresholds used in the real data. We then calculated how many voxels were overlapping in the two suprathreshold maps. The probability that the observed number of overlapping voxels in the real data $(k=$ 1141 ) would occur under this situation of random scattering of suprathreshold clusters was $p=0.003$, which indicates that it would be highly unlikely to observe these conjunction results if clusters in each map were randomly scattered across space. 


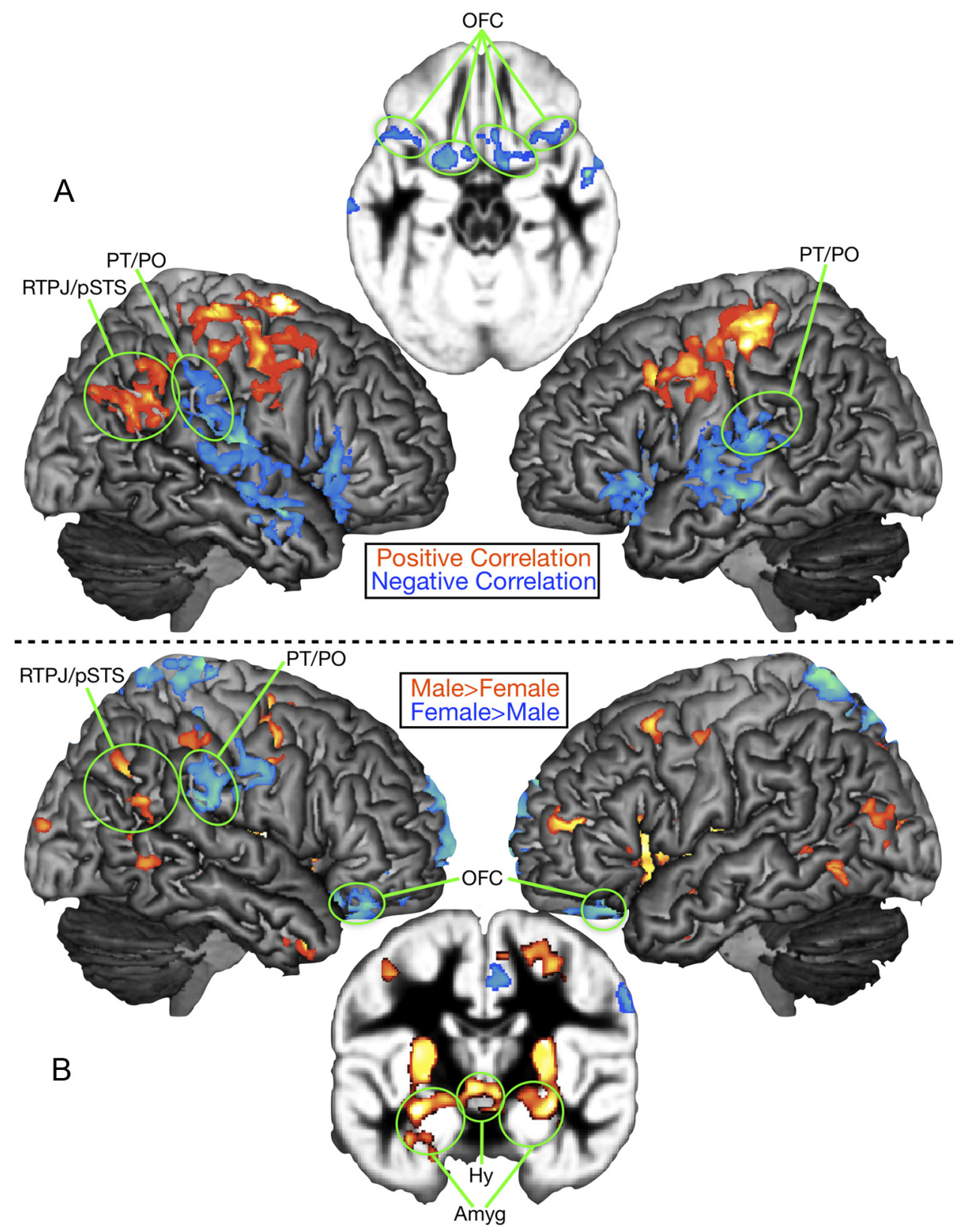

Figure 1. FT correlations with local GM volume. $\boldsymbol{A}$, Areas where FT predicts local gray matter volume. Red/orange voxels denote positive correlations; blue voxels denote negative correlations. $\boldsymbol{B}$, Areas of sexual dimorphism in local GM volume. Red/orange voxels denote a Male $>$ Female pattern; blue voxels denote a Female $>$ Male pattern.

\section{Magnitude of FT prediction of GM volume within sexually dimorphic regions}

Next, we estimated the magnitude of association between FT and local GM volume within sexually dimorphic voxels. First, we extracted mean GM volume from sexually dimorphic voxels in RTPJ/ pSTS, PT/PO, plOFC, PMd, bilateral amygdala, and hypothalamus. Because these voxels were identified in the NIH cohort, the ROI selection was independent of the test data from the FT cohort. We then used robust regression to compute a partial correlation between FT and local GM volume after controlling for age and total GM volume. FT was predictive of local GM volume in RTPJ/pSTS, $\mathrm{PT} / \mathrm{PO}$, and plOFC $\left(\mathrm{RTPJ} / \mathrm{pSTS}: r_{\text {partial }}=0.45, p=0.028\right.$; PT/PO: $r_{\text {partial }}=-0.47, p=0.013$; plOFC: $r_{\text {partial }}=-0.49, p=$ 0.006 ; Fig. $2 B-D)$, but not within $\operatorname{PMd}\left(r_{\text {partial }}=-0.0045, p=0.49\right)$. There was also no relationship between FT and local GM volume within sexually dimorphic voxels in the amygdala (Amyg) and hy- pothalamus (Hy) (left Amyg: $r_{\text {partial }}=0.01, p=0.48$; right Amyg: $r_{\text {partial }}=0.18, p=0.20$; left Hy: $r_{\text {partial }}=-0.20, p=0.18$; right Hy: $r_{\text {partial }}=-0.03, p=0.46$ ).

Because there was a slight difference in the proportion of right-handers across FT and NIH cohorts, we ran further analyses to assess whether the effect sizes of the FT correlations remain unchanged when the proportion of right-handers across the two cohorts was matched. To achieve this matching, we randomly removed two of the six non-right-handers in the FT cohort so that the percentage of right-handers matched that observed in the NIH cohort (85\%). These analyses were then done by iterating across all 15 possible combinations of removing two non-righthanders. The effect sizes for all regions remained similar to what was observed when analyzing the full FT cohort (RTPJ/pSTS: $r_{\text {partial }}$ range, $0.44-0.71$, all $p<0.05$; PT/PO: $r_{\text {partial }}$ range, $-0.41-0.48$, all $p<0.05$; plOFC: $r_{\text {partial }}$ range, $-0.39--0.58$, all 
A Males>Females \& FT Positive Correlation C Females $>$ Males \& FT Negative Correlation
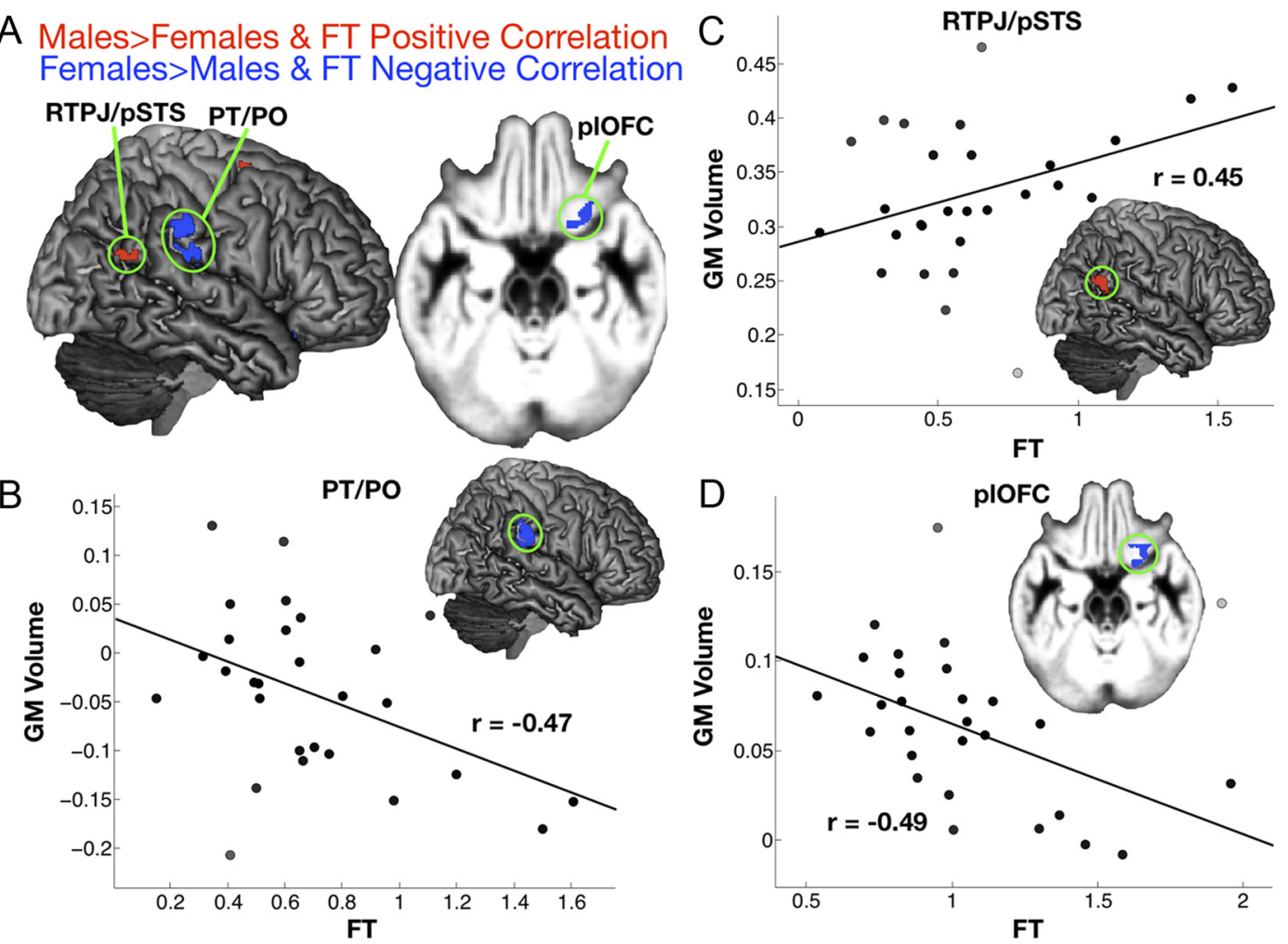

Figure 2. Overlap of FT-GM correlations and sexual dimorphism. $A$, Conjunction analysis overlap between sexual dimorphism and FT correlation in PT/P0, plOFC, and RTPJ/pSTS. Red voxels show overlap from the conjunction of FT positive correlation and Male $>$ Female; blue voxels show overlap from the conjunction of FT negative correlation and Female $>$ Male. $\boldsymbol{B}$, Scatterplot showing

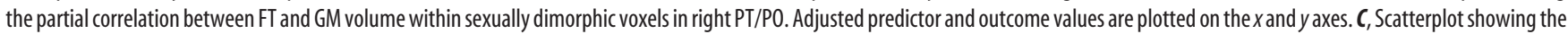
partial correlation between FT and GM volume within sexually dimorphic voxels in RTPJ/pSTS. Adjusted predictor and outcome values are plotted on the $x$ and $y$ axes. D, Scatterplot showing the partial correlation between FT and GM volume within sexually dimorphic voxels in right plOFC. Adjusted predictor and outcome values are plotted on the $x$ and $y$ axes.

$p<0.05$; PMd: $r_{\text {partial }}$ range, $-0.12-0.03$, all $p>0.05$; left Amyg: $r_{\text {partial }}$ range $=-0.05-0.07$, all $p>0.05$; right Amyg: $r_{\text {partial }}$ range, $0.09-0.21$, all $p>0.05$; left Hy: $r_{\text {partial }}$ range, $-0.22--0.09$, all $p>0.05$; right Hy: $r_{\text {partial }}$ range $=-0.10-0.07$, all $p>0.05$ ), demonstrating that proportion of right-handers in the FT cohort did not bias the results.

\section{Discussion}

Building on the long history of testing the organizing effects of early sex steroids on later sexual dimorphism in nonhuman species (Phoenix et al., 1959), the present study is the first to show that FT in humans influences specific brain regions that later develop in a sexually dimorphic fashion. Because our sample was restricted to males, the results cannot be easily explained by systematic differences between males and females in X-linked gene expression. Whether the observed effect of FT extends to females, or whether altogether different mechanisms affect variation in local gray matter volume in these regions, is still an open question. However, we can infer that sexual dimorphism in some parts of the human brain can come about through the early organizing influence that FT has on these regions within males. In other words, sexual dimorphism in these regions is expressed later in life because FT acts as a proximate signal in early development that influences early cellular processes (i.e., gene expression) that push later local gray matter throughout development in a direction that makes a difference between males and females more pronounced. Exactly what cellular processes FT influences early on to organize these brain regions for later sexually dimorphic development should be explored more in future research.
It is interesting to note that the regions we have identified here have also been observed to be sensitive to later testosterone and individual differences in androgen sensitivity. Paus and colleagues (2010) recently found that GM density in many regions, including $\mathrm{PT} / \mathrm{PO}$ and plOFC, were negatively related to current bioavailable testosterone levels and that this effect was more pronounced in those with a polymorphism of the androgen receptor gene $(A R)$ associated with enhanced androgen sensitivity. Similarly, Raznahan and colleagues (2010) observed longitudinal evidence that RTPJ exhibits a protracted period of increased cortical thickness in individuals with the $A R$ polymorphism associated with enhanced androgen sensitivity. These observations suggest that individual differences in sensitivity to androgens and both early organizational and later activational influences of androgen surges may be important for the expression of sexual dimorphism in these regions.

While we have highlighted three particular sexually dimorphic brain regions whose gray matter volume is related to FT levels, it is noteworthy that not all sexually dimorphic brain regions are related to FT or vice versa. For example, the amygdala and hypothalamus are known to be sexually dimorphic (Swaab and Fliers, 1985; Good et al., 2001), related to early and current androgen levels (Jacobson et al., 1981; Cooke et al., 1999), and, at least for the amygdala, related to sex chromosome differences and X-linked gene expression (Good et al., 2003). In the NIH dataset, these and other regions were sexually dimorphic in volume. With respect specifically to the amygdala, while we found a subregion of the amygdala that was positively related to FT, it did not overlap with the subregions that showed a Male $>$ Female sexual dimorphism. There are several possible explanations for the lack 
of a conjunction between sexual dimorphism and FT effects. First, it is possible that the difference in field strengths of the magnets in the FT cohort (3T) and NIH cohort (1.5T) could have led to biases for effects in one cohort but not the other (Tardif et al., 2010). Second, while the NIH cohort constitutes a random sample of the general population, the FT cohort is a selective subsample of the general population. The FT cohort is different in that they were more likely to be born to older mothers. Although all the participants in the FT cohort are considered typically developing children, there is still some possibility that some of the lack of correspondence between the data from these two datasets may reflect differences in maternal age of the two cohorts.

Finally, the lack of a conjunction between FT effects and sexual dimorphism for some brain regions may be due to a variety of other effects such as genetic differences from the sex chromosomes, interactions with later androgen surges, and other epigenetic influences. Regarding later androgen surges, interactions between FT and activational effects of current testosterone (CT) in puberty is one important area for focus in future work. In the current study, some of the older participants may have already entered puberty and this would create a potential difference in CT between older versus younger children. However, this potential effect covaries with age and the fact that age is a covariate in all analyses makes it less likely that this is a potential issue obscuring any FT influences observed in the current study. Nevertheless, in future studies, it will be important to test both FT and CT effects and any interactions they may have on local variation in human brain structure.

With regard to other epigenetic influences, one potential candidate is maternal influences on the fetal environment. Given the robust sex differences found in FT and the lack of any relationship between FT and testosterone in maternal blood, the fetus is generally considered the primary source of testosterone in the amniotic fluid (van de Beek et al., 2004). However, recent research suggests that the placenta is capable of synthesizing de novo androgens and estrogens (Escobar and Carr, 2011; Escobar et al., 2011). These types of placental influences should be investigated in future work to determine whether any such de novo sex steroids diffuse from the placenta into the amniotic fluid and how this may affect the fetus. If such sex steroids can enter into the amniotic fluid, it would constitute a further placental influence in addition to contributions made by the fetus itself. Teasing apart these more complex explanations in humans will be a challenge for future research.

The functions of the brain regions that are influenced by FT are especially interesting because of how they relate to previous literature on sex differences in cognition and behavior as well as behavioral correlations with FT. PT/PO overlaps with key language regions such as Wernicke's area and extends into parietal language areas known as Geschwind's Territory (Catani et al., 2005). We previously showed that increasing FT predicts smaller vocabulary at both 18 and 24 months; a result consistent with girls having larger vocabularies at this age (Lutchmaya et al., 2002a). Similarly, RTPJ/pSTS function has been associated with a host of social-cognitive and social-perceptual abilities such as mentalizing, social attention, eye-gaze processing, and empathy (Saxe and Powell, 2006; Decety and Lamm, 2007; Nummenmaa and Calder, 2009). We previously showed that increasing FT predicts less eye contact at 12 months (Lutchmaya et al., 2002b), decreased attributions of intentionality at 4 years (Knickmeyer et al., 2006), and decreased empathy at 8 years of age (Chapman et al., 2006). More recently, we showed that the extent to which current testosterone in adults impairs mentalizing performance is dependent on how much FT one was exposed to (van Honk et al., 2011). Thus, the current results suggest that the links between FT and behavior/cognition may be mediated by the influence that FT has in shaping brain structure underlying such functions. Future work should look specifically at whether FT effects on brain structure indeed mediate the links between FT and sexually dimorphic cognition and behavior.

These results may also have implications for neurodevelopmental conditions with skewed sex ratios (e.g., autism, developmental language, conduct disorder). Exactly how these results bear on such conditions remains to be seen. However, many neurodevelopmental conditions affect males more than females and many are characterized by deficits that are sexually dimorphic in the general population (e.g., language development, social cognition, self-regulation). These deficits have been hypothesized to be related to exposure to FT levels (Geschwind and Galaburda, 1985; Moffitt et al., 1998; Baron-Cohen, 2002; Rutter et al., 2004; Auyeung et al., 2009, 2010; Baron-Cohen et al., 2011). Furthermore, the sexually dimorphic brain regions we have found to be related to FT are all key brain areas for these particular conditions (Shaywitz et al., 1998; Rojas et al., 2002; Gervais et al., 2004; Pelphrey et al., 2005; Redcay and Courchesne, 2008; Passamonti et al., 2010; Lombardo et al., 2011; Raine et al., 2011). Thus, it will be important to look at FT and/or sex chromosome effects as potential mechanisms behind these conditions or how such mechanisms may affect one's risk for developing these types of conditions in early development (Baron-Cohen et al., 2011).

In sum, this study provides the first evidence that FT has an organizing effect on some sexually dimorphic areas of the human brain. Along with prior work on how FT influences behavior, this work highlights FT as an important developmental mechanism contributing to sex differences in neuroanatomy. Work on the organizing effects of FT on the developing human brain may be one important route for furthering our understanding of neurodevelopmental conditions that asymmetrically affect males more than females.

\section{Notes}

Supplemental material for this article is available at http:// autismresearchcentre.com/docs/lombardo_2011_jneurosci_Supplementary Table01.pdf. Supplemental Table 1: Areas where sexual dimorphism was observed in the NIH cohort. This material has not been peer reviewed.

\section{References}

Amunts K, Kedo O, Kindler M, Pieperhoff P, Mohlberg H, Shah NJ, Habel U, Schneider F, Zilles K (2005) Cytoarchitectonic mapping of the human amygdala, hippocampal region and entorhinal cortex: intersubject variability and probability maps. Anat Embryol (Berl) 210:343-352.

Arnold AP (2009) The organizational-activational hypothesis as the foundation for a unified theory of sexual differentiation of all mammalian tissues. Horm Behav 55:570-578.

Arnold AP, Breedlove SM (1985) Organizational and activational effects of sex steroids on brain and behavior: a reanalysis. Horm Behav 19:469-498.

Ashburner J (2007) A fast diffeomorphic image registration algorithm. Neuroimage 38:95-113.

Auyeung B, Baron-Cohen S, Ashwin E, Knickmeyer R, Taylor K, Hackett G (2009) Fetal testosterone and autistic traits. Br J Psychol 100:1-22.

Auyeung B, Taylor K, Hackett G, Baron-Cohen S (2010) Foetal testosterone and autistic traits in 18 to 24-month-old children. Mol Autism 1:11.

Baron-Cohen S (2002) The extreme male brain theory of autism. Trends Cogn Sci 6:248-254.

Baron-Cohen S, Lutchmaya S, Knickmeyer R (2004) Prenatal testosterone in mind. Cambridge: MIT.

Baron-Cohen S, Lombardo MV, Auyeung B, Ashwin E, Chakrabarti B, Knickmeyer R (2011) Why are autism spectrum conditions more prevalent in males? PLoS Biol 9:e1001081. 
Bergman K, Glover V, Sarkar P, Abbott DH, O'Connor TG (2010) In utero cortisol and testosterone exposure and fear reactivity in infancy. Horm Behav 57:306-312.

Brain Development Cooperative Group, Evans AC (2006) The NIH MRI study of normal brain development. Neuroimage 30:184-202.

Catani M, Jones DK, ffytche DH (2005) Perisylvian language networks of the human brain. Ann Neurol 57:8-16.

Chapman E, Baron-Cohen S, Auyeung B, Knickmeyer R, Taylor K, Hackett G (2006) Fetal testosterone and empathy: evidence from the empathy quotient (EQ) and the "reading the mind in the eyes" test. Soc Neurosci 1:135-148.

Cooke BM, Tabibnia G, Breedlove SM (1999) A brain sexual dimorphism controlled by adult circulating androgens. Proc Natl Acad Sci U S A 96:7538-7540.

Decety J, Lamm C (2007) The role of the right temporoparietal junction in social interaction: how low-level computational processes contribute to meta-cognition. Neuroscientist 13:580-593.

De Vries GJ, Rissman EF, Simerly RB, Yang LY, Scordalakes EM, Auger CJ, Swain A, Lovell-Badge R, Burgoyne PS, Arnold AP (2002) A model system for study of sex chromosome effects on sexually dimorphic neural and behavioral traits. J Neurosci 22:9005-9014.

Escobar JC, Carr BR (2011) The protein kinase a pathway regulates CYP17 expression and androgen production in the human placenta. J Clin Endocrinol Metab 96:2869-2873.

Escobar JC, Patel SS, Beshay VE, Suzuki T, Carr BR (2011) The human placenta expresses CYP17 and generates androgens de novo. J Clin Endocrinol Metab 96:1385-1392.

Forman SD, Cohen JD, Fitzgerald M, Eddy WF, Mintun MA, Noll DC (1995) Improved assessment of significant activation in functional magnetic resonance imaging (fMRI): use of a cluster-size threshold. Magn Reson Med 33:636-647.

Friston KJ (1997) Testing for anatomically specified regional effects. Hum Brain Mapp 5:133-136.

Gervais H, Belin P, Boddaert N, Leboyer M, Coez A, Sfaello I, Barthélémy C, Brunelle F, Samson Y, Zilbovicius M (2004) Abnormal cortical voice processing in autism. Nat Neurosci 7:801-802.

Geschwind N, Galaburda AM (1985) Cerebral lateralization: biological mechanisms, associations, and pathology. I. A hypothesis and a program for research. Arch Neurol 42:428-459.

Good CD, Johnsrude I, Ashburner J, Henson RN, Friston KJ, Frackowiak RS (2001) Cerebral asymmetry and the effects of sex and handedness on brain structure: a voxel-based morphometric analysis of 465 normal adult human brains. Neuroimage 14:685-700.

Good CD, Lawrence K, Thomas NS, Price CJ, Ashburner J, Friston KJ, Frackowiak RS, Oreland L, Skuse DH (2003) Dosage-sensitive X-linked locus influences the development of amygdala and orbitofrontal cortex, and fear recognition in humans. Brain 126:2431-2446.

Grimshaw GM, Sitarenios G, Finegan JA (1995) Mental rotation at 7 years: relations with prenatal testosterone levels and spatial play experiences. Brain Cogn 29:85-100.

Hines M (2004) Brain gender. New York: Oxford UP.

Jacobson CD, Csernus VJ, Shryne JE, Gorski RA (1981) The influence of gonadectomy, androgen exposure, or a gonadal graft in the neonatal rat on the volume of the sexually dimorphic nucleus of the preoptic area. J Neurosci 1:1142-1147.

Klein A, Andersson J, Ardekani BA, Ashburner J, Avants B, Chiang MC, Christensen GE, Collins DL, Gee J, Hellier P, Song JH, Jenkinson M, Lepage C, Rueckert D, Thompson P, Vercauteren T, Woods RP, Mann JJ, Parsey RV (2009) Evaluation of 14 nonlinear deformation algorithms applied to human brain MRI registration. Neuroimage 46:786-802.

Knickmeyer R, Baron-Cohen S, Raggatt P, Taylor K, Hackett G (2006) Fetal testosterone and empathy. Horm Behav 49:282-292.

Lombardo MV, Chakrabarti B, Bullmore ET, Baron-Cohen S (2011) Specialization of right temporo-parietal junction for mentalizing and its association with social impairments in autism. Neuroimage 56:1832-1838.

Lutchmaya S, Baron-Cohen S, Raggatt P (2002a) Foetal testosterone and vocabulary size in 18 - and 24-month-old infants. Infant Behav Dev 24:418-424.

Lutchmaya S, Baron-Cohen S, Raggatt P (2002b) Foetal testosterone and eye contact in 12-month-old human infants. Infant Behav Dev 25: 327-335.

McCarthy MM, Arnold AP (2011) Reframing sexual differentiation of the brain. Nat Neurosci 14:677-683.
McCarthy MM, Auger AP, Bale TL, De Vries GJ, Dunn GA, Forger NG, Murray EK, Nugent BM, Schwarz JM, Wilson ME (2009) The epigenetics of sex differences in the brain. J Neurosci 29:12815-12823.

Moffitt TE, Caspi A, Rutter M, Silva PA (1998) Sex differences in antisocial behavior. Cambridge: Cambridge UP.

Nichols T, Brett M, Andersson J, Wager T, Poline JB (2005) Valid conjunction inference with the minimum statistic. Neuroimage 25:653-660.

Nummenmaa L, Calder AJ (2009) Neural mechanisms of social attention. Trends Cogn Sci 13:135-143.

Oldfield RC (1971) The assessment and analysis of handedness: the Edinburgh inventory. Neuropsychologia 9:97-113.

Passamonti L, Fairchild G, Goodyer IM, Hurford G, Hagan CC, Rowe JB, Calder AJ (2010) Neural abnormalities in early-onset and adolescenceonset conduct disorder. Arch Gen Psychiatry 67:729-738.

Paus T, Nawaz-Khan I, Leonard G, Perron M, Pike GB, Pitiot A, Richer L, Susman E, Veillette S, Pausova Z (2010) Sexual dimorphism in the adolescent brain: role of testosterone and androgen receptor in global and local volumes of grey and white matter. Horm Behav 57:63-75.

Pelphrey KA, Morris JP, McCarthy G (2005) Neural basis of eye gaze processing deficits in autism. Brain 128:1038-1048.

Phoenix CH, Goy RW, Gerall AA, Young WC (1959) Organizing action of prenatally administered testosterone propionate on the tissues mediating mating behavior in the female guinea pig. Endocrinology 65:369-382.

Raine A, Yang Y, Narr KL, Toga AW (2011) Sex differences in orbitofrontal gray as a partial explanation for sex differences in antisocial personality. Mol Psychiatry 16:227-236.

Raznahan A, Lee Y, Stidd R, Long R, Greenstein D, Clasen L, Addington A, Gogtay N, Rapoport JL, Giedd JN (2010) Longitudinally mapping the influence of sex and androgen signaling on the dynamics of human cortical maturation in adolescence. Proc Natl Acad Sci U S A 107:16988-16993.

Redcay E, Courchesne E (2008) Deviant functional magnetic resonance imaging patterns of brain activity to speech in 2-3-year-old children with autism spectrum disorder. Biol Psychiatry 64:589-598.

Reinius B, Jazin E (2009) Prenatal sex differences in the human brain. Mol Psychiatry 14:987-989.

Rojas DC, Bawn SD, Benkers TL, Reite ML, Rogers SJ (2002) Smaller left hemisphere planum temporale in adults with autistic disorder. Neurosci Lett 328:237-240.

Rutter M, Caspi A, Fergusson D, Horwood LJ, Goodman R, Maughan B, Moffitt TE, Meltzer H, Carroll J (2004) Sex differences in developmental reading disability: new findings from 4 epidemiological studies. JAMA 291:2007-2012.

Sarkar P, Bergman K, Fisk NM, O'Connor TG, Glover V (2007) Amniotic fluid testosterone: relationship with cortisol and gestational age. Clin Endocrinol (Oxf) 67:743-747.

Saxe R, Powell LJ (2006) It's the thought that counts: specific brain regions for one component of theory of mind. Psychol Sci 17:692-699.

Shaywitz SE, Shaywitz BA, Pugh KR, Fulbright RK, Constable RT, Mencl WE, Shankweiler DP, Liberman AM, Skudlarski P, Fletcher JM, Katz L, Marchione KE, Lacadie C, Gatenby C, Gore JC (1998) Functional disruption in the organization of the brain for reading in dyslexia. Proc Natl Acad Sci U S A 95:2636-2641.

Swaab DF, Fliers E (1985) A sexually dimorphic nucleus in the human brain. Science 228:1112-1115.

Tardif CL, Collins DL, Pike GB (2010) Regional impact of field strength on voxel-based morphometry results. Hum Brain Mapp 31:943-957.

van de Beek C, Thijssen JH, Cohen-Kettenis PT, van Goozen SH, Buitelaar JK (2004) Relationships between sex hormones assessed in amniotic fluid, and maternal and umbilical cord serum: what is the best source of information to investigate the effects of fetal hormonal exposure? Horm Behav 46:663-669.

van Honk J, Schutter DJ, Bos PA, Kruijt AW, Lentjes EG, Baron-Cohen S (2011) Testosterone administration impairs cognitive empathy in women depending on second-to-fourth digit ratio. Proc Natl Acad Sci U S A 108:3448-3452.

Wager TD, Keller MC, Lacey SC, Jonides J (2005) Increased sensitivity in neuroimaging analyses using robust regression. Neuroimage 26:99-113.

Wilke M, Holland SK, Altaye M, Gaser C (2008) Template-O-matic: a toolbox for creating customized pediatric templates. Neuroimage 41:903-913.

Worsley KJ, Marrett S, Neelin P, Vandal AC, Friston KJ, Evans AC (1996) A unified statistical approach for determining significant signals in images of cerebral activation. Hum Brain Mapp 4:58-73. 